\title{
A model for studying cutaneous wound healing and resilience to aging: Ear punch biopsy in old mice
}

\author{
Zhou Jiang ${ }^{\mathrm{a}}$, Jessie Chen ${ }^{\mathrm{a}}$, Juan Wang, ${ }^{\mathrm{a}}$ Ruby Mangalindan ${ }^{\mathrm{a}}$, Lida Zhu, Warren C. Ladiges ${ }^{\mathrm{a},{ }^{*}}$ \\ ${ }^{a}$ Department of Comparative Medicine, School of Medicine, University of Washington, Seattle, WA, USA.
}

\begin{abstract}
Resilience to aging is a biological event that precedes age-related decline in physiological function and is defined as an organism's ability to respond to physical stress with increasing age. There is a need to identify factors that may predict resilience for enhancing and maintaining healthy aging. Older people often experience delayed wound healing beause of compromised tissue repair and immune response. Therefore preclincal models may be of value to investigate the relationship between cutaneous wound healing and resilience to aging. This brief report descibes an ear punch biopsy model of cutaneous wound healing in aging mice and shows that mice with biopsy ear wounds that heal more quickly have better cognition, increased strength and better running endurance later in life.
\end{abstract}

Keywords: Resilience to aging, wound healing, ear punch biopsy, aging mice

It is recognized that age-related decline in physiological function is preceded by resilience to aging, a biological event defined as an organism's ability to respond to physical stress with increasing age. However, there is a need to further characterize predictive factors of physical resilience for maintaining optimum age-related health. Wound healing in older people can be a health issue because of a delay in tissue repair and compromised immune response. Preclinical models are useful to develop better treatments, but also may be of interest to investigate correlation with systemic aging. However, current models are invasive and result in extensive tissue damage. Given that the ear of the mouse is readily accessible and easily manipulated, a small punch biopsy can create a wound with little peripheral damage. This report describes an ear punch biopsy model of cutaneous wound healing in aging mice to investigate the relation of resilience to healthy aging.

\section{Model description}

* Corresponding author: Warren Ladiges

Mailing address: Department of Comparative Medicine, School of Medicine, University of Washington, Seattle, WA, USA.

Email: wladiges@uw.edu

Received: 14 September 2020 / Accepted: 21 September 2020
C57BL/6 female mice, 20 months of age, were biopsied through the central area of both ears using a $2 \mathrm{~mm}$ dermal biopsy punch (Figure 1). Wound closure was measured on the day of the ear punch (Figure 2) and once a week for 5 weeks. Measurements were determined by flattening the ear with clear plastic plates, photographing the biopsy area, and applying the photograph to a base standard opening on a digital format where surface area in $\mathrm{mm}^{2}$ was determined by the elliptical formula: radius a (mm) $x$ radius $b(\mathrm{~mm}) \times 3.74(\square)$. Three months after the ear biopsy procedure, at 23 months of age, mice were tested for physiological performance using a spatial navigation task for learning [1], a rotating rod, grip strength, and distance ran over three days on a slanted, in-cage wheel [2].

As can be seen in Figure 2, there was no difference in closure area over five weeks between left and right ears, so the values from both ears could be pooled to increase the power of the analysis. The most significant increases in closure occurred from week 1 to week 2, where about 50 percent of the original opening had been closed in both ears.

A linear regression analysis of the decrease in open surface area over five weeks showed a surface area of 2.6 $\mathrm{mm}^{2}$ at two weeks. Therefore, mice with surface areas greater than $2.6 \mathrm{~mm}^{2}$ were considered slow healers, and mice with surface areas of $2.6 \mathrm{~mm}^{2}$ or less were considered fast healers. It was then possible to do correlation analysis with performance tests to see if fast healers aligned with increased performance. As can be seen in 


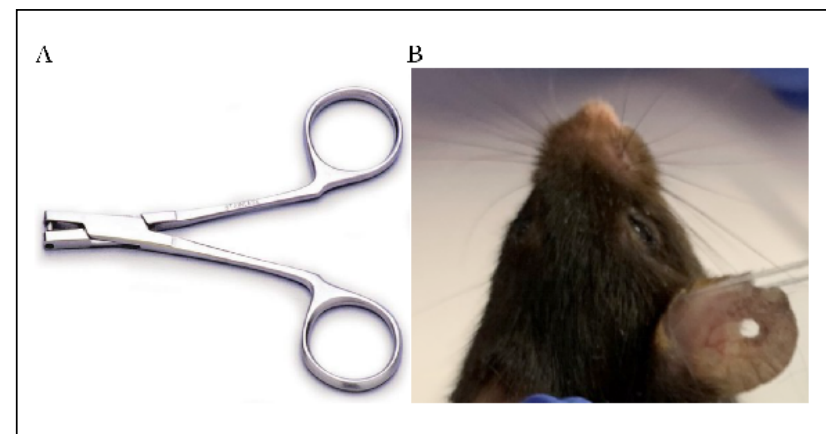

Figure 1. Ear biopsy procedure. A. A $2 \mathrm{~mm}$ ear punch biopsy instrument (VWR) was used for B. creating a through and through biopsy opening in the central part of each ear.

Figure 3, fast healers were able to find an escape hole more quickly, had increased grip strength, and ran further over three days than slow healers.Increased performance in aging mice of the same gender (all were females) with faster wound closure suggests factors other than age and genetic background may be playing a role in resilience to aging. Additional studies of fast and slow healers would

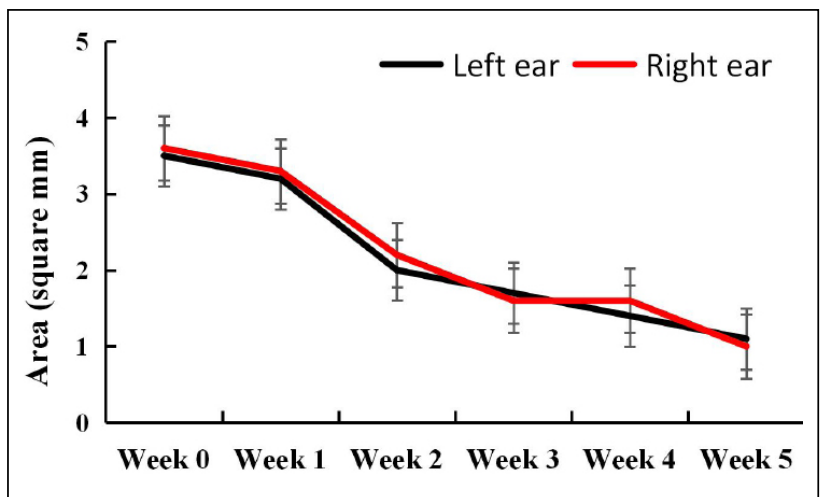

Figure 2. C57BL/6 female mice, 20 months of age, had close to a 50 percent closure after two weeks following an ear punch biopsy. $N=20$ mice. There was no difference in closure in either ear at any time point up to five weeks.

be of interest to determine molecular pathways involved. In addition, the ear punch biopsy procedure would be a useful relatively noninvasive model for studying how cutaneous wound healing is related to systemic internal organ aging.

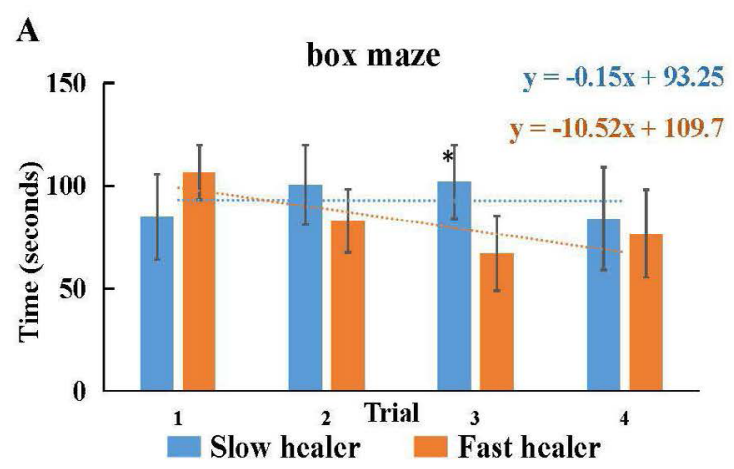

C

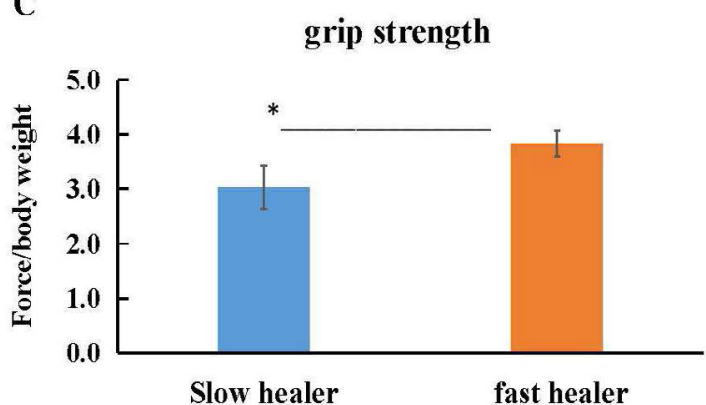

$\mathbf{B}$

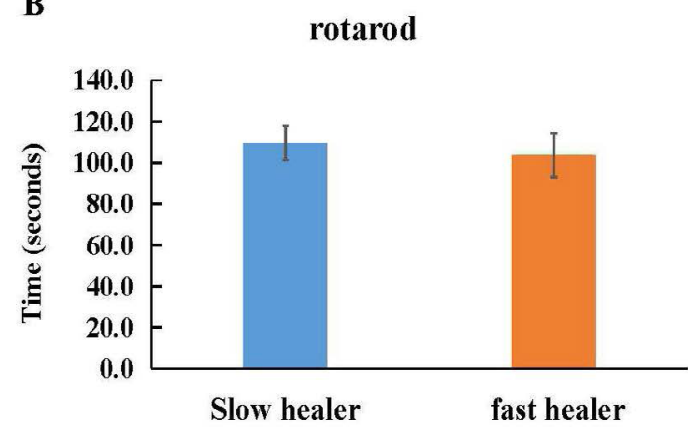

D

running wheel

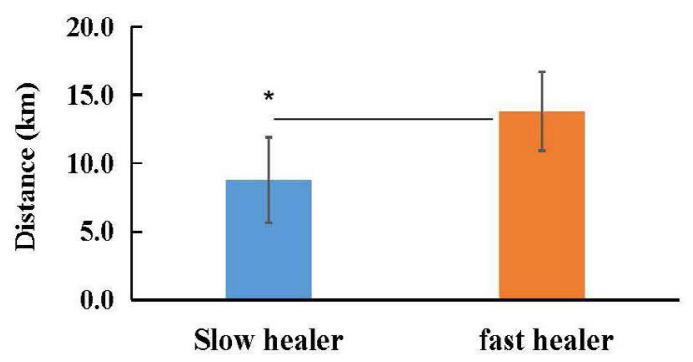

Figure 3. Mice that had increased closure of an ear punch biopsy after two weeks (fast healers) were: A. Better at finding an escape hole in a spatial navigation learning task (box maze), B. Had similar times staying on a rotating rod, C. Had increased grip strength, and D. Ran further over three days on a slanted wheel, compared to mice with a decreased ear biopsy closure (slow healers). All performance tests were conducted three months after the ear biopsy. $N=8-10$ mice per cohort. * $P \leq 0.05$. 


\section{Declarations}

Acknowledgments: Supported by a grant from the National Institute on Aging, R56 AG058543 (W. Ladiges, PI)

Conflict of Interest: Warren Ladiges is a member of the Editorial Board of Aging Pathobiology and Therapeutics. All authors declare no conflict of interest and were not involved in the journal's review or desicions related to this manuscript.

\section{References}

1. Darvas M, Mukherjee K, Lee A, et al. A Novel One-Day Learning Procedure for Mice. Current Protocols in Mouse Biology, 2020, 10(1): e68.

2. Goh J, Ladiges W. Voluntary wheel running in mice. Current protocols in mouse biology, 2015, 5(4): 283-290.

Cite this article as: Zhou J, Jessie C, Juan W, et al. A model for studying cutaneous wound healing and resilience to aging: Ear punch biopsy in old mice.[J]. Aging Pathobiology and Therapeutics, 2020, 2(3): 173-175 